\title{
Battling tumors with magnetic nanotherapeutics and hyperthermia: turning up the heat
}

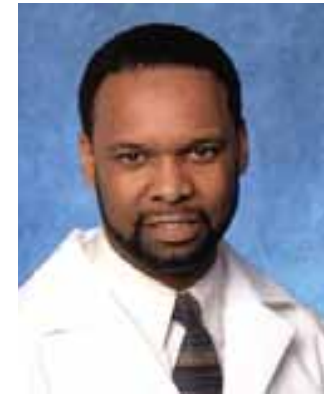

Robert B Campbell

$N$ ortheastern U niversity, D epartment of

Pharmaceutical Sciences, 360 $H$ untington Avenue, Boston, M A 02115, USA

Tel.: +1 6173733091

Fax: +1 6173738886

E-mail: r.campbell@neu.edu

future part of $\mathrm{fsg}$ “...the suc cess a chieved with different na notec hnology-

treatment forms in the clinic continues to support their use in combination with other treatment moda lities."

Using nanotherapeutics in combination with magnetic fields is an attractive therapeutic approach. M agnetic fields are used alone or in combination with other treatment modalities, such as surgery, chemotherapy and hyperthermia. Phase I clinical reports confirm that the use of alternating magnetic fields (AMF) is safe enough for clinical application, although optimization for the best clinical results is still needed. $\mathrm{N}$ anomedicine might represent the greatest opportunity to apply magnetic fields for cancer therapy; however, we must also understand, which properties of early formulations require additional characterization and optimization. The development of magnetite cationic liposomes (M CLs) is an example of a relatively new nanotreatment with clinical potential based on early formulation studies and preclinical reports. M CLs are taken up by target cells when administered by local injection; when target cells internalize magnetite they become highly susceptible to hyperthermia-inducing effects. When M CLs are loaded with chemotherapeutic agents, cancer cells are even more susceptible to selective heat induction (or hyperthermia). An example is seen with 4-S-cysteaminylphenol (4-S-CAP) incorporated in M CLs used alone and in combination with hyperthermia against malignant melanoma. I discuss these data as well as the implications of 'turning up the heat' in cancer therapy.

Antitumor drug targeting is an attractive but challenging area of cancer research. The delivery of clinically relevant concentrations of drugs to tumor targets is hindered by many physiological obstacles [1,2]. Several known barriers are tumor hypertension, disorganized blood-vessel arrangement, long travel distances for therapeutics on entering the tumor matrix and high interstitial collagen content. All of these factors impede interstitial drug transport. A strategy used to improve delivery and overall treatment effectiveness has been to use agents that limit the role of particular barrier effects long enough to produce significant therapeutic gains [1]. For example, preexposing tumors to agents that lower interstitial fluid pressure improves tumor response; however, the responses vary according to the particular agent, dose and schedule used. Success with this approach is often linked to improved vascular flow (or perfusion) and vascular remodeling effects.

Another approach is to exploit enhanced tumor-vascular permeability by developing nanoparticles that are small enough to penetrate the tumor interstitial matrix but still large enough to avoid passive accumulation in normal healthy tissues. This approach is generally referred to as passive drug targeting and has demonstrated significant benefit over administration of chemotherapeutic agents alone. Such benefits include enhanced treatment effectiveness and reduced toxicity and, when polyethyleneglycol (PEG) is included in the formulation, other advantages, such as favorable plasma-clearance profiles, are observed [3]. Even though the effects of PEG were considered revolutionary for the liposome field, the lack of tumor-specific uptake still remains an important issue to overcome. M ajor improvements in terms of target specificity will probably improve cytotoxic drug effects, reduce doserelated toxicity and thus permit the use of more aggressive treatment schedules.

'Nanomedic ine might represent the grea test opportunity to apply magnetic fields for cancer thera py...'

More active-targeting methods have been introduced to improve passive-targeting approaches. The coupling of antibodies (directed against the functional expression of cellular targets in vivo) to the surface of drugcarrier molecules is used to maximize delivery to tumor targets while minimizing uptake by healthy tissues. Immunoliposome technology is one such example. When applied, a significantly higher fraction of the injected dose/g of 
target tissue is recovered [4]. Although these discoveries have been critically important to the field of drug targeting, we continue to seek out new strategies to improve target specificity for various applications.

Although our fundamental knowledge of how tumors can be exploited for therapeutic gain has been developing, other promising observations have been revisited for cancer therapy. For example, early studies revealed that the application of heat just a few degrees above physiological temperature was sufficient for the treatment of malignant tumors. Researchers began developing strategies to apply heat to cancer-positive lymph nodes when it was discovered that cancer spreads to lymph nodes by tumor emboli. In accord with this reasoning, scientists investigated the effect of localizing fine magnetic particles in lymph nodes followed by 'selective heat induction' [5]. The approach was successful in eradicating undetectable disease. This was considered an important clinical breakthrough with future medical implications. H owever, the magnetic particles used in this study were developed over five decades ago and today the field of nanomedicine has created new opportunities to improve target specificity.

'... early studies revealed that the application of heat just a few degrees above physiological temperature was sufficient for the treatment of malignant tumors.'

Since the discovery that high temperatures can be used to abolish tumor growth and thus limit the progression of cancer disease, selective heat induction (an early form of hyperthermia) has received a considerable amount of attention. $H$ yperthermia is generally applied as one of three different treatment strategies: local hyperthermia $(\mathrm{LH})$, regional hyperthermia $(\mathrm{RH})$ or whole body hyperthermia (WBH). LH restricts the application of heat directly to the tumor site; $\mathrm{RH}$ is often used to treat an entire organ or limb and $\mathrm{WBH}$ applies heat to the entire body. $\mathrm{H}$ eat is generally applied by microwaves, high-frequency radiowaves, special radiant-heat systems and even high-energy magnets when placed over the entire tumor area. N ow, of course, the major goal of hyperthermia in cancer treatment is to eradicate tumor growth but this is not achieved easily without major risks to host tissues. For example, external tissue burning is a common side effect of treatment. $O$ ne solution is to maximize toxic effects to areas of malignancy by confining heat effects to the volume of the tumor. Another approach is to inject magnetic compounds directly into the tumor area before the application of heat. In this case, tumors show an increase in actual blood supply when temperatures reach between 40 and $41^{\circ} \mathrm{C}$; however, when applied at relatively high temperatures $\left(>42^{\circ} \mathrm{C}\right)$, the result is a compromised microcirculation and eventual tumor-vascular collapse. It is thus reasonable to hypothesize that highly selective uptake of magnetic material by intended target cells would restrict hyperthermic effects to diseased areas and thus spare injury to surrounding tissues.

Chemotherapy can be administered in combination with hyperthermia, given the ability to load both magnetite and chemotherapeutic agents simultaneously in liposomes [6]. In the $\mathrm{M}$ arch 2007 issue of Cancer Science, Ito and colleagues showed promising results using hyperthermia and 4-S-CAP-loaded magnetite cationic liposomes to treat malignant melanoma [7]. The agent 4-S-CAP is a substrate of melanoma tyrosinase and is used in this study to induce selective killing of melanocytes and melanoma cells [8]. Preliminary studies in vitro compared the effects of 4-S-CAP treatment alone, hyperthermia at $42.5^{\circ} \mathrm{C}$ and as a combination treatment approach. Cytotoxicity studies revealed the following effects:

4-S-CAP < hyperthermia < 4-S-CAP + hyperthermia

The researchers showed uptake of 4-S-CAP magnetite nanoparticles by B16 cells and provide evidence of their beneficial use in vitro. When B16 cells were placed under the influence of AM F irradiation, the cells containing magnetite nanoparticles generated more heat when compared with controls ( 0 pg magnetite/cell). The magnetite nanoparticles enabled the sustained rapid heating of cancer cells at temperatures known to cause hyperthermia-inducing effects. Control cells never reached effective hyperthermia-inducing temperatures, suggesting the ability of magnetite nanoparticles to accelerate the normal rate of heating, which is a desirable feature of this nanosystem.

When 4-S-CAP/MCL $(100 \mu \mathrm{M})$ was injected by the intratumoral route, the whole body of the tumor-bearing mouse was placed under the influence of an AM F. It took only 3 min for the tumor temperature to reach $45^{\circ} \mathrm{C}$, which was held for $30 \mathrm{~min}$; only marginal increases in temperature were reported for the rectum (the normal tissue control). As noted 
earlier, a commonly associated side effect of hyperthermia is burning of healthy tissue; no unwanted burn effects were observed with the combined 4-S-CAP/MCL hyperthermia-treatment approach. The authors note similarities between 4-S-CAP/MCL- and MCL-induced hyperthermia, suggesting the importance of magnetite to achieve rapid heat effects and cationic-charge potential of nanoparticles for improved retention in tumors.

'...for hea ting devic es to ga in a ccess to the highly proliferating tumor rim without

causing damage to the host, either na notechnology or the technology for the application of heat needs improvement.'

An in vivo hyperthermic treatment study showed an additive therapeutic effect of 4-S-C AP/M CLs and hyperthermia that was significantly more effective than 4-S-CAP/M C Ls or AM F irradiation alone. The combined treatment approach prevented progressive tumor growth and the formation of pulmonary metastasis and resulted in massive necrosis and hemorrhagic effects. Interestingly, complete tumor regression of melanoma was not observed in this study, which was probably owing to suboptimal temperatures to induce hyperthermic effects. In this study, the temperature was set at approximately $42^{\circ} \mathrm{C}$, lower than temperatures reported previously to induce hyperthermic effects. The temperatures used in this study provided scientists with a window to evaluate the antitumor activity of 4-S-CAP, which would be overshadowed by hyperthermia-inducing effects at higher temperatures.

$\mathrm{N}$ ew-generation cationic liposomes have been evaluated extensively for delivery of genes to mammalian cells in vitro and in vivo and have been considered only within the last 6 years for delivery of small cytotoxic molecules. The agent 4-S-CAP/MCL is now part of the emerging nanomedicine landscape and several interesting features of the systems applied by Ito and colleagues should be discussed. First, the MCLs were injected by the intratumor route and so physiological barriers, such as high interstitial tumor pressures, are overcome easily. Substantial doses are delivered to tumors prior to the application of heat. In contrast to intratumorinjection strategies, systemic administration can reach more distant, inaccessible or even undetectable cancer disease; however, relatively nonspecific uptake owing to intravenous injections of magnetite could cause rapid hyperthermia-inducing effects in unwanted tissues. Dose-related toxicity is not a problem for intratumor injections because, under these conditions, the bulk of the drug is in the tumor, not in normal tissues. Also, when more conventional chemotherapeutic agents are loaded in cationic liposomes, preferential accumulation in target tissues is absolutely essential given the relatively nonselective action of these agents. This is not a limitation for 4-S-CAP/M CL sowing to the enhanced selective action of 4-S-CAP against melanoma cells [8]. Finally, most of the clinical experiences with hyperthermia (including studies investigated by Ito and colleagues) have been performed with superficial and subcutaneous tumors. The technology is not well developed for deep-seated tumors. It is known that the threshold of thermal damage for normal tissues is actually similar for tumors. Therefore, for heating devices to gain access to the highly proliferating tumor rim without causing damage to the host, either nanotechnology or the technology for the application of heat needs improvement.

$\mathrm{H}$ yperthermia is mostly considered an adjunct to other therapeutic approaches, such as surgery and chemotherapy, and has yet to approach the level of a first-line clinical approach in the USA. N onetheless, the success achieved with different nanotechnology-treatment forms in the clinic continues to support their use in combination with other treatment modalities. Future studies combining chemotherapy and hyperthermia should continue to exploit unique cellular features for therapeutic gain and, regardless of the combination treatment approach, it should be optimized for individualized treatment.

Financial \& competing interests disclosure

The authors have no relevant affiliations or financial involvement with any organization or entity with a financial interest in or financial conflict with the subject matter or materials discussed in the manuscript. This includes employment, consultancies, honoraria, stock ownership or options, expert testimony, grants or patents received or pending or royalties.

$\mathrm{N}$ o writing assistance was utilized in the production of this manuscript. 


\section{Bibliography}

1. Campbell RB: Tumor physiology and delivery of nanopharmaceuticals. Anticancer Agents M ed. Chem. 6, 501-510 (2006).

2. Jain RK: The next frontier of molecular medicine: delivery of therapeutics. $\mathrm{N}$ at. M ed. 4, 655-657 (1998).

3. M ori A, Klivanov AL, Torchilin VP, $H$ uang $L$ : Influence of the steric barrier activity of amphipathic poly(ethyleneglycol) and ganglioside G M 1 on the circulation time of liposomes and on the target binding of immunoliposomes in vivo. FEBS Lett. 284, 263-266 (1991).
4. Allen TM : Ligand-targeted therapeutics in anticancer therapy. $N$ at. Rev. Cancer 2 , 750-763 (2002).

5. Gilchrist RK, M edal R, Shorey WD, $H$ anselman RC, Parrott JC, Taylor CB: Selective inductive heating of lymph nodes. Ann. Surgery 146, 596-606 (1957).

6. D andamudiS, Campbell RB: The drug loading, cytotoxicity and tumor vascular targeting characteristics of magnetite in magnetic drug targeting. Biomaterials 28(31), 4673-4683 (2007).
7. Ito A, Fujioka M, Yoshida T et al.: 4-S-cysteaminylphenol-loaded magnetite cationic liposomes for combination therapy of hyperthermia with chemotherapy against malignant melanoma. Cancer Sci. 98, 424-430 (2007).

8. M inamitsuji $Y$, Toyofuku K, Sugiyama $S$, Yamada K, Jimbow K : Sulfur containing tyrosine analogs can cause selective melanocytotoxicity involving tyrosinasemediated apoptosis. J. Invest. Dermatol. Symp. Proc. 4, 130-136 (1999). 